\title{
Prognostic implications of cell division cycle protein 45 expression in hepatocellular carcinoma
}

\author{
Chen Yang Equal first author, 1, 2 , shufang Xie ${ }^{\text {Equal first author, } 2,3}{ }^{\text {, yi wu }}{ }^{4}$, Guoqing Ru ${ }^{5}$, Xianglei He ${ }^{5}$, Hong-yin Pan ${ }^{6}$, Shibing

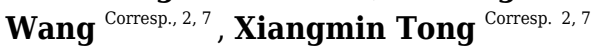 \\ 1 Department of Clinical Medicine, Qingdao University, Qingdao, China \\ 2 Molecular diagnosis laboratory, Zhejiang Provincial People's Hospital, Hangzhou, China \\ 3 The Second Clinical Medical College, Zhejiang Chinese Medical University, Hangzhou, China \\ 4 Phase I clinical research center, Zhejiang Provincial People's Hospital, Hangzhou, China \\ 5 Department of Pathology, Zhejiang Provincial People's Hospital, Hangzhou, China \\ 6 Department of Infectious diseases, Zhejiang Provincial People's Hospital, Hangzhou, China \\ 7 The Key Laboratory of Tumor Molecular Diagnosis and Individualized Medicine of Zhejiang Province, Zhejiang Provincial People's Hospital, Hangzhou, \\ China
}

Corresponding Authors: Shibing Wang, Xiangmin Tong

Email address: wangshibing@hmc.edu.cn, tongxiangmin@163.com

Background. The overall prognosis of hepatocellular carcinoma (HCC) is poor and novel prognostic biomarkers might better monitor the progression of HCC . Cell division cycle protein 45 ( CDC45 ) plays a key role in DNA replication and considered to be involved in tumorigenesis. This study investigated CDC45 expression in tumour tissues and defined its prognostic value in HCC patients. Methods. We used immunohistochemistry ( IHC ) staining to examine the expression of CDC45 in tumour tissue specimens and compare them with adjacent normal tissue specimens using a constructed tissue microarray ( TMA ) and analyzed how clinical features are related to HCC prognosis. Functional enrichment analyses were used to describe significantly involved hallmark pathways of differentially expressed genes ( DEGs, which were screened out according to the high or low expression of CDC45 in tumour tissues). Results. Our findings showed that the proteome expression of CDC45 was evidently downregulated in HCC tissues compared with matched normal tissues $(P<0.0001)$. Although we did not find any differences in terms of vascular invasion, metastasis, lymphatic infiltration, or Edmondson grade between patients with high and low CDC45 expression, low CDC45 expression was significantly correlated with microvascular invasion ( $P=0.046)$. Multivariate analysis indicated that $C D C 45$ expression $(P=0.035)$ was an independent prognostic factor for the overall survival (OS ) rate of HCC patients. Patients with CDC45 expression was positively correlated with OS rates among HCC patients $(P<0.05)$. Functional annotations indicated that $C D C 45$ is involved in the most significant pathways, including the cell cycle, DNA replication, chemical carcinogenesis and drug metabolism-cytochrome P450 pathways. Discussion. Our findings showed that Peer) reviewing PDF | (2020:09:52495:1:2:NEW 11 Dec 2020) 
low proteomic level of CDC45 was associated with a poor prognosis in HCC patients, indicating that CDC45 might be a novel prognostic marker 
1 Prognostic implications of cell division cycle protein 45 expression in hepatocellular

2 carcinoma

3

5 Chen Yang ${ }^{1,2}$ ๆ, Shufang Xie ${ }^{2,3}$ ฯ,, $\mathrm{Yi} \mathrm{Wu}^{4}$, Guoqing $\mathrm{Ru}^{5}$, Xianglei $\mathrm{He}^{5}$, Hongyin Pan ${ }^{6}$, Shibing

6 Wang $^{2,7^{*}}$, Xiangmin Tong ${ }^{2,7^{*}}$

8 1. Department of Clinical Medicine, Qingdao University, Qingdao, P. R. China

9 2. Molecular diagnosis laboratory, Zhejiang Provincial People's Hospital, People's Hospital of

10 Hangzhou Medical College, Hangzhou, Zhejiang, P. R. China

11 3. The Second Clinical Medical College, Zhejiang Chinese Medical University, Hangzhou, P.

4. Phase I clinical research center, Zhejiang Provincial People's Hospital, People's Hospital of Hangzhou Medical College, Hangzhou, P. R. China

5. Department of Pathology, Zhejiang Provincial People's Hospital, People's Hospital of Hangzhou Medical College, Hangzhou, P. R. China

6. Department of Infectious diseases, Zhejiang Provincial People's Hospital, People's Hospital

7. The Key Laboratory of Tumor Molecular Diagnosis and Individualized Medicine of Zhejiang College), Hangzhou, P. R. China

\footnotetext{
* Corresponding author
} 
24 Email :wangshibing@hmc.edu.cn(SBW) ; tongxiangmin@163(XMT)

25 These authors contributed equally to this work.

\section{Abstract}

Background. The overall prognosis of hepatocellular carcinoma (HCC) is poor and novel prognostic biomarkers might better monitor the progression of HCC. Cell division cycle protein 45 (CDC45) plays a key role in DNA replication and considered to be involved in tumorigenesis. This study investigated CDC45 expression in tumour tissues and defined its prognostic value in HCC patients.

Methods. We used immunohistochemistry (IHC) staining to examine the expression of CDC45 in tumour tissue specimens and compare them with adjacent normal tissue specimens using a constructed tissue microarray (TMA) and analyzed how clinical features are related to HCC prognosis

. Functional enrichment analyses were used to describe significantly involved hallmark pathways of differentially expressed genes (DEGs, which were screened out according to the high or low expression of CDC45 in tumour tissues).

Results. Our findings showed that the proteome expression of CDC45 was evidently downregulated in HCC tissues compared with matched normal tissues $(\mathrm{P}<0.0001)$. Although we

41 did not find any differences in terms of vascular invasion, metastasis, lymphatic infiltration, or Edmondson grade between patients with high and low CDC45 expression, low CDC45 expression was significantly correlated with microvascular invasion $(\mathrm{P}=0.046)$. Multivariate analysis indicated that $\mathrm{CDC} 45$ expression $(\mathrm{P}=0.035)$ was an independent prognostic factor for the overall survival (OS) rate of HCC patients. Patients with CDC45 expression was positively 
46

47

correlated with OS rates among HCC patients $(\mathrm{P}<0.05)$. Functional annotations indicated that CDC45 is involved in the most significant pathways, including the cell cycle, DNA replication, chemical carcinogenesis and drug metabolism-cytochrome $\mathrm{P} 450$ pathways.

Discussion. Our findings showed that low proteomic level of CDC45 was associated with a poor prognosis in $\mathrm{HCC}$ patients, indicating that CDC45 might be a novel prognostic marker

Keyword: Cell division cycle protein 45, hepatocellular carcinoma, biomarker, bioinformatics

\section{Introduction}

Hepatocellular carcinoma (HCC) is the most common type of primary liver cancer and ranks as the fourth leading cause of cancer mortality all over the world(F et al. 2018). In the last few years, its incidence has sharply increased(C et al. 2018). Although some curative therapies, including hepatic resection, liver transplantation and the application of targeted drugs, can prolong survival for HCC, the 5-year OS for HCC patients is quite disappointing(A et al. 2020;

JK et al. 2018). In addition, the heterogeneity of liver cancer hinders choosing the optimal treatment for patients(LQ et al. 2019),(B et al. 2020). The biomarkers that can primely predict the prognosis may guide treatment in HCC patients and improve HCC clinical outcomes. Nevertheless, the specificity and sensitivity of current biomarkers still need to be improved(JC \& A 2020). Therefore, it is particularly critical to identify effective biomarkers for the diagnosis and prognosis of patients with liver cancer, which might provide the opportunity to use targeted drugs and immune modulators earlier in the disease course.

CDC45 is an integral component of the CDC45-MCM2-7-GINS (CMG) helicase complex, which plays a crucial role in DNA replication, especially in the initiation stagey(AC et al. 2016; R \& HP 2009).Even it is still unclear whether CDC45 plays any role in the repair pathways in 
87

mammalian cells, CDC45 plays an important biological role in maintaining the genomic stability(A et al. 2013),(A \& V 2012).Furthermore, it has been indicated that CDC45 is important for the response to replication stress by interacting with ssDNA(I \& DL 2013) and recruiting Rad53(G et al. 2019).Recently,CDC45 expression was identified to associate with tumorigenesis and was useful for tumour prognosis. Previous studies have shown high CDC45 expression in human cancer-derived cell lines, including breast carcinoma, cervix carcinoma and acute lymphoblastic leukaemia(S et al. 2007). Moreover, it was demonstrated that the upregulation of CDC45 in papillary thyroid cancer is correlated with a more advanced tumour stage( $\mathrm{J}$ et al. 2017). In nonsmall cell lung cancer, CDC45 can promote the growth of tumour cells and was defined as an oncogene(J et al. 2019). However, rare studies have investigated the relationship between CDC45 expression and predictive value in HCC.

In the present study, we examined the protein expression level of CDC45 in HCC by comparing tumours with neighbouring normal tissues in tissue microarrays (TMAs). Then, a larger sample size was used to investigate the association of CDC45 expression and clinical characteristics of patients with HCC. Additionally, we analysed the potential biological interaction network and prognostic value of CDC45. We hypothesized that the possible antioncogenic activity of CDC45 may influence the HCC patients' prognosis. Our results may uncover a feasible therapeutic target and provide insights into the molecular mechanisms of CDC45.

\section{Materials and methods}


89

90

91

92

93

94

95

96

97

98

99

100

101

102

103

104

105

106

107

108

109

110

\section{Patient characteristics and construction of the tissue microarray}

TMAs from $321 \mathrm{HCC}$ patients who took curative hepatectomy for primary HCC in Zhejiang Provincial People's Hospital between 2008 and 2015 were used for validation. The ethics committee of Zhejiang Provincial People's Hospital granted Ethical approval to carry out the study within its facilities (Ethical Application Ref:2019KY232). All patients did not receive radiotherapy or chemotherapy before surgery. The whole group of patients were informed and signed informed consent forms. Total survival was calculated from the date of surgery to the end of follow-up (December 2016) or the time of death. In total, 321 HCC patients in our study presented with a mean age of $56.9 \pm 11.4$ years (range, $25.0-90.0$ years), with $60(18.7 \%)$ females and $261(81.3 \%)$ males (Table 1$)$. At the time of the primary diagnosis, $52.1 \%$ of the patients presented with a tumour diameter less than $5 \mathrm{~cm}, 47.9 \%$ with a tumour diameter greater than or equal to $5 \mathrm{~cm}$. A total of $63.2 \%$ had an Edmondson grade of I/II, and $36.8 \%$ had grade III. In addition, the numbers of patients with or without metastasis were $288(91.4 \%)$ and 27 (8.6\%), respectively. The median OS was 23.0 months (range, 1.0-74.0). Other clinical characteristics are displayed in Table 1.

\section{Immunohistochemical staining}

IHC was performed using standard techniques. Briefly, 5- $\mu \mathrm{m}$ paraffin-embedded TMA sections were dewaxed using xylene (sinopharm, China)and rehydrated in graded alcohols(sinopharm, China). Next, blocked endogenous peroxidase with 3\% hydrogen peroxide (sinopharm, China). Antigen retrieval was accomplished via adding $10 \mathrm{mM}$ citrate buffer ( $\mathrm{pH}$ 6.0) (sinopharm, China) to the TMA sections and putting them into a high-pressure cooker. Then, the TMA 
111 sections were incubated with 1\% bovine serum albumin (BSA, Sigma, German) for 20 min to

112 reduce nonspecific protein binding. The recombinant rabbit monoclonal CDC45 antibody (1:50;

113 HuaBio, Hangzhou, China) were next used to treat the TMA sections at room temperature for

$1141 \mathrm{~h}$. They were then rinsed with phosphate-buffered saline (PBS, HuaBio, Hangzhou, China ) and

115 incubated with biotinylated secondary antibody (MXB, Fuzhou, China) for 30 min at room

116 temperature. Subsequently, the TMA sections were stained with DAB chromogen (Gene Tech,

117 Shanghai, China), counterstained with Mayer's haematoxylin (HuaBio, Hangzhou, China),

118 dehydrated with gradient alcohol and xylene, and mounted on slides. Finally, the TMA sections

119 were viewed under a Nikon light microscope.

120

121 Cell culture

122 Human normal liver cell line LO2 and hepatocellular carcinoma cell lines (HCC-LM3, MHCC-

97H, Huh7 and Hep3b) were purchased from the ATCC and cultured in Dulbecco's modified

Eagle's medium (DMEM, Gibco, USA)) containing 10\% fetal bovine serum (Gibco, USA) and $1 \%$ penicillin/streptomycin (Beyotime, China) in a humid atmosphere with $5 \% \mathrm{CO} 2$ at $37{ }^{\circ} \mathrm{C}$.

\section{Western blot and antibodies}

Cells were washed with PBS (Beyotime, China) twice. Next, RIPA buffer were used to lyse the cells. Thereafter, BCA Protein Quantitation Assay (Beyotime, China) was used to detected the protein concentration of the cells. Proteins were resolved using 10\% SDS-PAGE and then 
131

132

133

134

135

136

137

138

139

140

141

142

143

144

145

146

147

148

149

150

151

transferred onto a nitrocellulose membrane. After block with 5\% non-fat milk for $1 \mathrm{~h}$ at room temperature, the membranes were then incubated with the primary antibodies at $4{ }^{\circ} \mathrm{C}$ overnight.

After that, TBST was used to wash the membranes three times and subsequently incubated with secondary antibodies (anti-rabbit IgG or anti-mouse IgG) for $1 \mathrm{~h}$ at room temperature. Next, membranes were washed with TBST three times. The targeted proteins were identified using the ECL (Thermofisher, USA). CDC45 and ACTIN antibodies used in study were purchased from HuaBio (ET1701-71, China) and Abcam (ab8227) respectively.

\section{Assessment of CDC45 expression}

The Densito Quant software in Quant Center was applied to automatically identify and set the areas on the tissue sections as follows: dark brown as strong positive, tan as moderate positive, blue (nucleus) as negative, and light yellow as weak positive. Then, each tissue point was identified and analysed to find the areas of strong positive, medium positive, weak positive and negative (unit: pixel); then, the percentage of positive staining and the histochemistry score (Hscore) were calculated. The median H-score (median $=7.44)$ was selected as the cut-off value to classify the level of CDC45 expression. H-scores $<7.44$ were considered as low CDC45 expression, and H-scores $>=7.44$ were used to define tumours with high CDC45 expression.

\section{Bioinformatics analysis of GSE76427}

We used gene expression omnibus (GEO, http:/www.ncbi.nlm.nih.gov/geo/) database, an international public platform including high-throughput gene expression and other functional genomics datasets for the research community(T et al. 2013). We selected the GSE76427(OV et 
152 al. 2018) dataset and downloaded the original CEL files as well as the platform files to explore

153 CDC45 expression and its potential mechanisms by means of R software. Specifically, according

154 to the median value of the CDC45 gene among HCC tissues, samples were divided into two

155 groups including high or low expression. Then, the adjusted $\mathrm{p}$-value $<0.05$ and $|\log 2 \mathrm{FC}|>1.5$

156 were used as cut-off criteria to screen differentially expressed genes (DEGs) between the two

157 groups of samples. Subsequently, we conducted Gene Ontology (GO) and Kyoto Encyclopedia

158 of Genes and Genomes (KEGG) pathway enrichment analyses with DEGs. In addition, the

159 protein-protein interaction (PPI) network of DEGs was constructed with a online search tool for

160 the retrieval of interacting genes (STRING; http://string-db.org), and to analyse the functional

161 interactions between proteins (an interaction with a combined score $>0.9$ was considered

162 statistically significant), which were then visualized using Cytoscape. Moreover, we verified

163 important modules via using the molecular complex detection (MCODE) app plugin of

164 Cytoscape.

165

166

167

168

169

170

171

172

173

174

\section{Statistical analysis}

All data were analysed using SPSS 22.0 software (SPSS Inc., Chicago, IL, USA). The univariate and multivariate hazards were determined by Cox proportional hazards model. Differences between HCC and noncancerous tissues were examined by paired t-test. The correlation results between the clinicopathological parameters and the CDC45 expression were investigated by the chi-square analysis. The comparation of CDC45 expression among different cell lines was examined by t-test. We used Kaplan-Meier analysis to estimate survival, and any differences in survival were evaluated with a stratified log-rank test. Generally, $\mathrm{P}<0.05$ was considered statistically significant. 
175

176

177

178

179

180

181

182

183

184

185

186

187

188

189

190

191

192

193

\section{Results}

Our research consisted of 3 stages. Firstly, we assessed protein expression of CDC45 in our TMA. Then we investigated the association of CDC45 expression with clinical characteristics and its prognostic value in HCC. In the third stage, we screened the significantly involved DEGs of CDC45 and performed corresponding functional annotations using DEGs.

\section{Validation of CDC45 expression in HCC and adjacent normal tissues}

We use TMA to detected the protein expression levels. IHC staining was used to evaluate the CDC45 protein expression levels in HCC tumour samples and matched normal tissues from 56 HCC patients (Fig 1a). CDC45 staining was mainly distributed in the cytoplasm. Compared to side normal tissues, the protein expression of CDC45 was significantly decreased in HCC tissues $(\mathrm{P}<0.0001$, Fig 1b)

\section{Association of CDC45 expression with clinical characteristics}

We then explored the correlation between CDC45 expression levels and the clinicopathological parameters of HCC patients. Among the clinicopathological features, CDC45 expression was positively related with tumour size $(\mathrm{P}=0.034)$ and microvascular invasion $(\mathrm{P}=0.046)$. However, there was no significant correlation between CDC45 expression and other clinical parameters (gender, age, tumour number, hepatitis B surface antigen (HBS), Edmondson grade, vascular invasion, cirrhosis and metastasis, $\mathrm{P}>0.05$; Table 1). These findings suggested that low CDC45 expression is associated with worse overall disease condition. 


\section{Clinical values of CDC45 expression in the prognosis of $\mathrm{HCC}$}

The Kaplan-Meier survival curve revealed that CDC45 expression was evidently associated with OS in HCC patients ( $\mathrm{P}=0.019$; Fig 1c). In all HCC patients, the OS was shorter in patients with low CDC45 expression than those who with high CDC45 expression. Additionally, Cox regression was used to analyse the prognostic factors of HCC. Our univariate Cox regression revealed that tumour size $(\mathrm{P}=0.007)$, metastasis $(\mathrm{P}<0.001)$, microvascular invasion $(\mathrm{P}=0.022)$, Edmondson grade $(\mathrm{P}<0.001)$ and vessel invasion $(\mathrm{P}=0.011)$ were independent prognostic factors in patients with HCC. A multivariate Cox regression analysis revealed that distant metastases $(\mathrm{P}<0.001)$ and Edmondson grade $(\mathrm{P}=0.013)$ were independent prognostic factors for patients with HCC (Table 2). Consistent with the results of the Kaplan-Meier analysis, both the univariate $(\mathrm{P}=0.022)$ and multivariate $(\mathrm{P}=0.035)$ Cox regression analysis indicated that $\mathrm{CDC} 45$ expression was closely associated with the prognosis of HCC.

\section{Identification of DEGs and functional annotations as well as predicted signalling pathways}

To further study the potential biological function of CDC45 in HCC, the genes from the GSE76427 dataset were divided into two groups based on the median value of CDC45 expression to screen the DEGs between the low and high CDC45 expression groups. The screening criteria are discussed in the Materials and methods section). As shown in Figure 2a, there were 233 upregulated and 167 downregulated DEGs. Specifically, the top 20 significant DEGs with positive and negative correlations were shown in Figure $2 b$ with a heat map. Subsequently, 400 involved DEGs were subjected to conduct GO annotation and KEGG pathway analyses. And top $30 \mathrm{GO}$ terms with the highest gene enrichment are shown in Figure 2c. Those related genes were significantly involved in eukaryote division included organelle 
216 fission, nuclear division, chromosome segregation, mitotic nuclear division, and were markedly

217 involved in the regulation of the cell cycle phase transition and DNA replication. Moreover, the

218 KEGG analysis showed that most of the involved significant pathways contained the cell cycle,

219 DNA replication, drug metabolism and metabolism of xenobiotics by cytochrome P450,

220 chemical carcinogenesis, meiosis and fatty acid degradation signalling (Fig 2d). The specified

221 functional annotations, KEGG analysis information and percentage of each term are shown in 222 additional file 1 .

223 To further explore the interplay among the DEGs, we used the STRING online database and

224 Cytoscape software to construct a PPI network. As illustrated in Figure 3a, the network includes

225

226

227

228

229

230

231

232

233

234

235

236

237

238

400 nodes and 1,622 edges. The nodes with positive correlation with CDC45 expression are shown as yellow, nodes with negative correlation are shown as blue, and CDC45 itself is purple. PPI network was then carried out clustering analysis by Cytotype MCODE, the top three significant modules were selected according to the degree of importance. Module 1 contains 43 nodes and 683 edges (Fig 3b), which means that CDC45 interacts is closely related those 43 proteins. module 2 contains 13 nodes and 39 edges (Fig 3c); and module 3 contains 19 nodes and 58 edges (Fig 3d). Submodule analysis information is shown in additional file 2.

\section{Discussion}

CDC45 is a key component of the CMG complex and necessary for the initiation and elongation of chromosomal DNA replication(AC et al. 2016),(P et al. 1998). In recent years, several reports have shown that its expression is related to the occurrence and development of tumours. It has been reported that CDC45 overexpression was observed in lung cancer and was considered a novel tumour-associated antigen (TAA) that might be a useful target for lung cancer immunotherapy(Y et al. 2011). Furthermore, $\mathrm{Hu} \mathrm{Y}$ et al. verified that the upregulated CDC45 
239

240

241

242

243

244

245

246

247

248

249

250

251

252

253

254

255

256

257

258

259

260

261

expression in colorectal cancer patients was associated with poor prognosis(Y et al. 2019).

A previous study also revealed that the CDC45 protein level is consistently higher in various

kinds of human tumour cells than in normal cells so that was identified as a

proliferation-associated antigen(S et al. 2007).

However, in our study, we found that CDC45 expression at the protein level was downregulated in HCC by detecting the expression of CDC45 in tumour tissues and matched adjacent normal liver tissues from $56 \mathrm{HCC}$ patients. Although there are fewer samples in this study, this pairing analysis of tumour and adjacent normal liver tissues seems to be more convincing. Similarly, WB results showed that CDC45 expression was lower in HCC-LM3 cells, MHCC-97H and Huh7 cells than nomal liver LO2 cells (FigureS1). Moreover, our results demonstrated that high protein expression of CDC45 negatively correlated with tumour size and metastasis, which revealed that decreased expression of CDC45 may promote cancer progression. Importantly, the Kaplan-Meier survival analysis indicated that HCC patients with a low expression of CDC45 have an inferior prognosis than patients with a high expression of CDC45. To further analyse the possible causes, we screened for DEGs related to CDC45 expression from the GEO database and conducted GO and KEGG analyses. Our results showed that the genes mostly enriched in the GO terms included organelle fission, nuclear division, chromosome segregation, mitotic nuclear division, regulation of cell cycle phase transition, regulation of mitotic cell cycle phase transition, nuclear chromosome segregation, sister chromatid segregation, DNA replication, and cell cycle G1/S phase transition. Furthermore, the KEGG analysis showed that most of the involved significant pathways included the cell cycle, DNA replication, drug metabolism-cytochrome P450, metabolism of xenobiotics by cytochrome P450, and chemical carcinogenesis. Therefore, we speculated that the correlation between CDC45 and HCC might be 
262 related to these functions.

263 Actually, it is common for the same gene to play different roles in various tumours. Typically,

264 Sprouty2, which inhibits the growth and spread of tumour in breast cancer, prostate cancer and

265 liver cancer, may contributes to the promotion of colorectal cancer metastasis(Zhang et al. 2016).

266 Although it has been reported that CDC45 is a proliferative marker in some cancers, there are

267 also reports stressed that the high expression of CDC45 may lead to severe replication pressure,

268 subsequently occurring S-phase arrest, and eventually lead to cellular apoptotic cell death,

269 inhibiting the degradation of CDC45 might be a promising way to combat cancer(Köhler et al.

270 2016; Zhang et al. 2016). In addition to the sustained proliferation, the escape from apoptosis,

271 genomic instability, and DNA replication stress are also considered hallmarks of human cancers

272 and are closely related to tumorigenesis and progression(M \& TD 2015).Therefore, high

273 expression of CDC45 induced DNA stress of cancer cells, leading to cell death might explain the

274 poor prognosis of low CDC45 expression in HCC patients.

275 This study has several limitations. First, we only conduct a single center study in our hospital,

276 number of patients may bring some limitations, Secondly, even though a series of functional

277 annotations and enrichment analyses were performed, the underlying mechanisms of signalling

278 pathways in HCC remain ambiguous. Although the research has some limitations, we are

279 committed to provide some basis for the biological function of CDC45 in liver cancer. In the

280 future, we may expand the sample size and even conduct multi center research for better verify.

281 In addition, to explore the detailed mechanism between CDC45 and carcinogenesis as well as

282 reveal the mechanism of CDC45 in other cancers.

\section{Conclusions}

284 In conclusion, our research revealed the crucial role of CDC45 in patients with HCC. Decreased 
285 CDC45 protein expression correlated with the progression and poor prognosis in $\mathrm{HCC}$,

286 indicating that CDC45 is a valuable promising prognostic biomarker and might be a potential

287 treatment target in HCC. Further studies are required to analyse its specific biological function in 288 HCC.

289

Additional Information and Declarations

\section{Ethics approval and consent to participate}

The Ethics approval and consent to participate of the current study was approved and consented by the ethics committee of Zhejiang Provincial People's Hospital.

\section{Competing Interests}

296 The authors declare that they have no competing interests.

297

298

299

300

301

302

303

304

\section{Author Contributions}

All authors have significant contributions to the conception of the work. Chen Yang and Shufang Xie analyzed statistics and drafted the manuscript. Yi Wu, Guoqing Ru ,Xianglei He provided the clinical data and revised manuscript. Hongyin Pan, Shibing Wang, Xiangmin Tong participated clarification of data and provided final approval of the submitted manuscript. All authors read and approved the final manuscript.

\section{Data Availability}

All data generated or analyzed during this study are included in this published article. 


\section{Funding}

307

308

309

310

311

312

313

314

315

316

317

318

319

320

This study was supported by the Zhejiang Medical Technology Plan Project (No.WKJ-ZJ-1709, 2020KY052), Zhejiang Provincial Natural Science Foundation of China (No. LY19H160037, LGF18H160025, LY17H160062, 2017C33116).

\section{Acknowledgements}

We would like to thank American Journal Experts(http://www.aje.com/) that editing the English version of this manuscript.

\section{Supporting information}

S1 File. KEGG analysis information and percentage of each term

S2 File. GO analysis information and percentage of each term

S3 File. PPI and submodule analysis information

\section{References}

A A, JC Z, A C, and V C. 2013. DNA replication and homologous recombination factors: acting together to maintain genome stability. Chromosoma 122:401-413. 10.1007/s00412-013-0411-3

A E, and V C. 2012. Mechanisms of replication fork protection: a safeguard for genome stability. Critical reviews in biochemistry and molecular biology 47:222-235. 10.3109/10409238.2012.655374

A K, SS F, B H, RM R, GB K, DD L, CB T, F A, AD T, A H, EC V, KJ H, WC C, N V, M H, MH L, MH N, ML M, AN L, CA C, C M, M G, B A, JF M, DL S, CM J, J B, AW H, JC H, J K, MA Z, TL N, A R, ML K, TM F, D M, RW B, and VG A. 2020. Liver Transplantation Outcomes in a U.S. Multicenter Cohort of 789 Patients with Hepatocellular Carcinoma Presenting Beyond Milan Criteria. Hepatology (Baltimore, Md). 
10.1002/hep.31210

AC S, V S, V C, and L P. 2016. Structure of human Cdc45 and implications for CMG helicase function. Nature communications 7:11638. 10.1038/ncomms11638

B L, AJ C, C V-M, SN M-F, N A, X C, ME A, J vF, I L, D DA, K A, SA L, GC F, T G-L, P R, A S, SC W, MI F, SP H, G G, D S, EE S, R S, M S, JM L, S T, G S, and A V. 2020. Intratumoral heterogeneity and clonal evolution in liver cancer. Nature communications 11:291. 10.1038/s41467-019-14050-z

C A, T M, V DC, R H, M M, M N, A B, M V, CJ J, J E, OJ O, G AES, WQ C, S E, G E, CA S, A M, RR W, O V, GH L, J A, HK W, and MP C. 2018. Global surveillance of trends in cancer survival 2000-14 (CONCORD3): analysis of individual records for 37513025 patients diagnosed with one of 18 cancers from 322 population-based registries in 71 countries. Lancet (London, England) 391:1023-1075. 10.1016/s01406736(17)33326-3

F B, J F, I S, RL S, LA T, and A J. 2018. Global cancer statistics 2018: GLOBOCAN estimates of incidence and mortality worldwide for 36 cancers in 185 countries. CA: a cancer journal for clinicians 68:394-424. $10.3322 /$ caac. 21492

G C, AC K, D M, and P Z. 2019. Helicase Subunit Cdc45 Targets the Checkpoint Kinase Rad53 to Both Replication Initiation and Elongation Complexes after Fork Stalling. Molecular cell 73:562-573.e563. 10.1016/j.molcel.2018.11.025

I B, and DL K. 2013. Cdc45 protein-single-stranded DNA interaction is important for stalling the helicase during replication stress. The Journal of biological chemistry 288:7550-7563. 10.1074/jbc.M112.440941

J H, Y L, Z L, Y C, S S, S M, Y L, R Z, N L, S Z, C L, X W, N S, and J H. 2019. Analysis of functional hub genes identifies CDC45 as an oncogene in non-small cell lung cancer - a short report. Cellular oncology (Dordrecht) 42:571-578. 10.1007/s13402-019-00438-y

J S, R S, S Z, X L, S L, H B, and X M. 2017. Cell division cycle 45 promotes papillary thyroid cancer progression via regulating cell cycle. Tumour biology : the journal of the International Society for Oncodevelopmental Biology and Medicine 39:1010428317705342. 10.1177/1010428317705342

JC N, and A V. 2020. Biomarkers for hepatobiliary cancers. Hepatology (Baltimore, Md). 10.1002/hep.31175

JK H, LM K, RS F, CB S, MM A, LR R, AX Z, MH M, and JA M. 2018. AASLD guidelines for the treatment of hepatocellular carcinoma. Hepatology (Baltimore, Md) 67:358-380. 10.1002/hep.29086

Köhler C, Koalick D, Fabricius A, Parplys A, Borgmann K, Pospiech H, and Grosse FJCc. 2016. Cdc45 is limiting for replication initiation in humans. 15:974-985. 10.1080/15384101.2016.1152424

LQ D, LH P, LJ M, DB L, S Z, SZ L, JH R, HW Z, SX Y, SJ X, M C, FF X, FQ L, WH L, C Y, LY L, YJ W, XY W, DM G, H Z, HM Y, J W, SD Z, XD W, Y C, J Z, J F, K W, and Q G. 2019. Heterogeneous immunogenomic features and distinct escape mechanisms in multifocal hepatocellular carcinoma. Journal of hepatology. 10.1016/j.jhep.2019.12.014

M M, and TD H. 2015. DNA replication stress as a hallmark of cancer. Annual review of pathology 10:425-448. 10.1146/annurev-pathol-012414-040424

OV G, SP Y, R I, M S, HK L, KH L, PK C, and VA K. 2018. Tumor-adjacent tissue co-expression profile analysis reveals pro-oncogenic ribosomal gene signature for prognosis of resectable hepatocellular carcinoma. Molecular oncology 12:89-113. 10.1002/1878-0261.12153

P S, KC T, R Y, Z H, S W, and A D. 1998. The human homolog of Saccharomyces cerevisiae CDC45. The Journal of biological chemistry 273:18205-18209. 10.1074/jbc.273.29.18205 
370

371

372

373

374

375

376

377

378

379

380

381

382

383

384

385
R B, and HP N. 2009. Regulation of Cdc45 in the cell cycle and after DNA damage. Biochemical Society transactions 37:926-930. 10.1042/bst0370926

S P, C B, J S, HP N, and F G. 2007. Human Cdc45 is a proliferation-associated antigen. The FEBS journal 274:36693684. 10.1111/j.1742-4658.2007.05900.x

T B, SE W, P L, C E, IF K, M T, KA M, KH P, PM S, M H, A Y, H L, N Z, CL R, N S, S D, and A S. 2013. NCBI GEO: archive for functional genomics data sets--update. Nucleic acids research 41:D991-995. 10.1093/nar/gks1193

Y H, L W, Z L, Z W, M S, S W, and G W. 2019. Potential Prognostic and Diagnostic Values of CDC6, CDC45, ORC6 and SNHG7 in Colorectal Cancer. OncoTargets and therapy 12:11609-11621. 10.2147/ott.S231941

Y T, K I, S S, A I, M I, Y H, K S, T M, Y D, T T, T I, H N, Y N, H K, and Y N. 2011. A novel tumor-associated antigen, cell division cycle 45-like can induce cytotoxic T-lymphocytes reactive to tumor cells. Cancer science 102:697-705. 10.1111/j.1349-7006.2011.01865.x

Zhang Q, Wei T, Shim K, Wright K, Xu K, Palka-Hamblin H, Jurkevich A, and Khare SJO. 2016. Atypical role of sprouty in colorectal cancer: sprouty repression inhibits epithelial-mesenchymal transition. 35:3151-3162. 10.1038/onc.2015.365 


\section{Table 1 (on next page)}

Association between the expression of CDC45 expression and clinical characteristics of HCC patients 
1 Table 1. Association between the expression of CDC45 expression and clinical characteristics

2 of HCC patients

\begin{tabular}{|c|c|c|c|c|}
\hline \multirow{2}{*}{$\begin{array}{l}\text { Clinicopathological } \\
\text { variables }\end{array}$} & \multirow[t]{2}{*}{$\mathrm{n}(\%)$} & CDC45 & & \multirow[t]{2}{*}{ P-value } \\
\hline & & Low () & High () & \\
\hline Sex & & & & 0.213 \\
\hline Male & $261(81)$ & 129 & 132 & \\
\hline Female & 60 (19) & 35 & 25 & \\
\hline Age (years) & & & & 0.544 \\
\hline$<55$ & $124(39)$ & 66 & 58 & \\
\hline$>=55$ & $197(61)$ & 98 & 99 & \\
\hline Size $(\mathrm{cm})$ & & & & 0.034 \\
\hline$<5$ & $\begin{array}{l}163 \\
(52.1)\end{array}$ & 75 & 88 & \\
\hline$>=5$ & $\begin{array}{l}150 \\
(47.9)\end{array}$ & 87 & 63 & \\
\hline Tumour number & & & & 0.797 \\
\hline Single & $264(82)$ & 134 & 130 & \\
\hline Multiple & $57(18)$ & 30 & 27 & \\
\hline Edmondson grade & & & & 0.299 \\
\hline I - II & $\begin{array}{l}199 \\
(63.2)\end{array}$ & 96 & 103 & \\
\hline III & $\begin{array}{l}116 \\
(36.8)\end{array}$ & 63 & 53 & \\
\hline Metastasis & & & & 0.306 \\
\hline MO & $288(91)$ & 147 & 141 & \\
\hline M1 & $27(9)$ & 11 & 16 & \\
\hline Vessel invasion & & & & 0.169 \\
\hline Absence & $120(50)$ & 62 & 58 & \\
\hline Presence & $119(50)$ & 72 & 47 & \\
\hline $\begin{array}{l}\text { Microvascular } \\
\text { invasion }\end{array}$ & & & & 0.046 \\
\hline Negative & $\begin{array}{l}133 \\
(57.6)\end{array}$ & 68 & 65 & \\
\hline Positive & $\begin{array}{l}98 \\
(42.4)\end{array}$ & 63 & 35 & \\
\hline
\end{tabular}


HBs antigen

Negative

Positive

Cirrhosis

Negative

Positive
59 (19) $28 \quad 31$

$256(81) \quad 132 \quad 124$

$106(33) \quad 54 \quad 52$

$215(67) \quad 110 \quad 105$
0.57

0.971

2

3 
Table 2 (on next page)

Univariate and multivariate Cox regression survival analyses 
1 Table 2. Univariate and multivariate Cox regression survival analyses

\begin{tabular}{|c|c|c|c|c|c|c|}
\hline \multirow[b]{2}{*}{ Parameters } & \multicolumn{3}{|c|}{ Univariate analyses } & \multicolumn{3}{|c|}{ Multivariate analyses } \\
\hline & \multicolumn{2}{|c|}{$\begin{array}{l}\text { Hazard ratio }(95 \% \\
\mathrm{Cl})\end{array}$} & P-value & $\begin{array}{l}\text { Hazard } \\
(95 \% \mathrm{Cl})\end{array}$ & ratio & \multirow{2}{*}{$\begin{array}{l}\mathrm{P} \text { - } \\
\text { value } \\
\mathbf{0 . 0 3 5}\end{array}$} \\
\hline $\begin{array}{l}\text { CDC45 } \\
\text { expression }\end{array}$ & $\begin{array}{l}0.558 \\
0.920)\end{array}$ & $(0.338-$ & 0.022 & $\begin{array}{l}0.509 \\
0.957)\end{array}$ & $(0.271-$ & \\
\hline Size & $\begin{array}{l}1.965 \\
3.213)\end{array}$ & $(1.201-$ & 0.007 & $\begin{array}{r}1.157 \\
2.177)\end{array}$ & (0.614- & 0.652 \\
\hline Metastasis & $\begin{array}{l}5.075 \\
9.636)\end{array}$ & (2.673- & 0.000 & $\begin{array}{l}6.533 \\
14.635)\end{array}$ & (2.916- & 0.000 \\
\hline $\begin{array}{l}\text { Microvascular } \\
\text { invasion }\end{array}$ & $\begin{array}{l}1.879 \\
3.219)\end{array}$ & (1.097- & 0.022 & $\begin{array}{l}1.015 \\
2.753)\end{array}$ & (0.374- & 0.977 \\
\hline $\begin{array}{l}\text { Vessel } \\
\text { invasion }\end{array}$ & $\begin{array}{l}2.035 \\
3.525)\end{array}$ & $(1.174-$ & 0.011 & $\begin{array}{l}1.636 \\
4.440)\end{array}$ & (0.602- & 0.334 \\
\hline $\begin{array}{l}\text { Edmondson } \\
\text { grade }\end{array}$ & $\begin{array}{l}2.696 \\
4.392)\end{array}$ & $(1.655-$ & 0.000 & $\begin{array}{l}2.082 \\
3.715)\end{array}$ & $(1.166-$ & 0.013 \\
\hline
\end{tabular}

$2 \mathrm{Cl}$, confidence interval. 


\section{Box 1 (on next page)}

CDC45 protein expression and prognostic implications in the TMA of HCC

(A) IHC staining indicated significantly downregulated CDC45 expression in HCC tissues comparing to the adjacent normal liver tissues in the TMA. (B) The differential CDC45 protein expression in 56 paired tumour and non-tumorous liver tissues $(p<0.0001)$. (C) Kaplan-Meier survival analysis indicated that low CDC45 expression was indicated a shorter OS $(p=0.019)$. 


\section{Scheme 1}

CDC45 protein expression and prognostic implications in the TMA of HCC

(A) IHC staining indicated significantly downregulated CDC45 expression in HCC tissues

comparing to the adjacent normal liver tissues in the TMA. (B) The differential CDC45 protein expression in 56 paired tumour and non-tumorous liver tissues $(p<0.0001)$. (C)

Kaplan-Meier survival analysis indicated that low CDC45 expression was indicated a shorter OS $(p=0.019)$. 
A

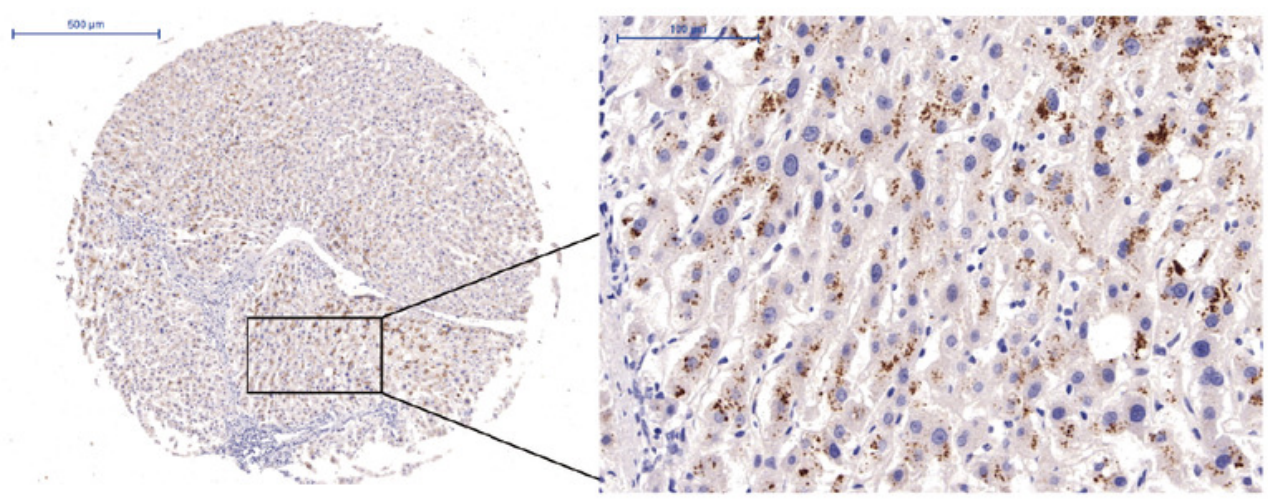

Normal

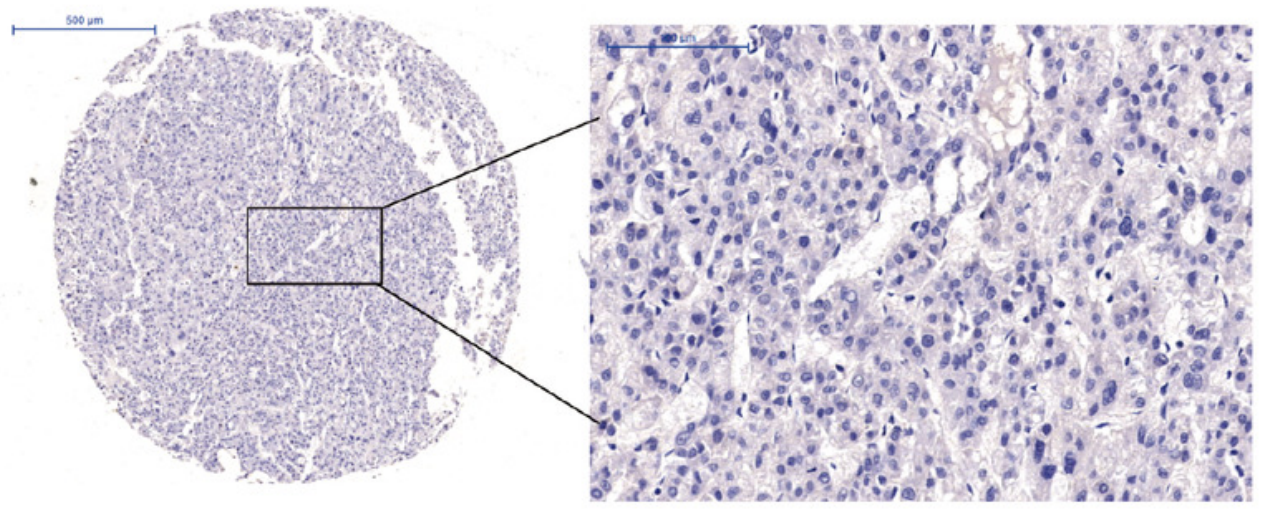

\section{Tumor}

B

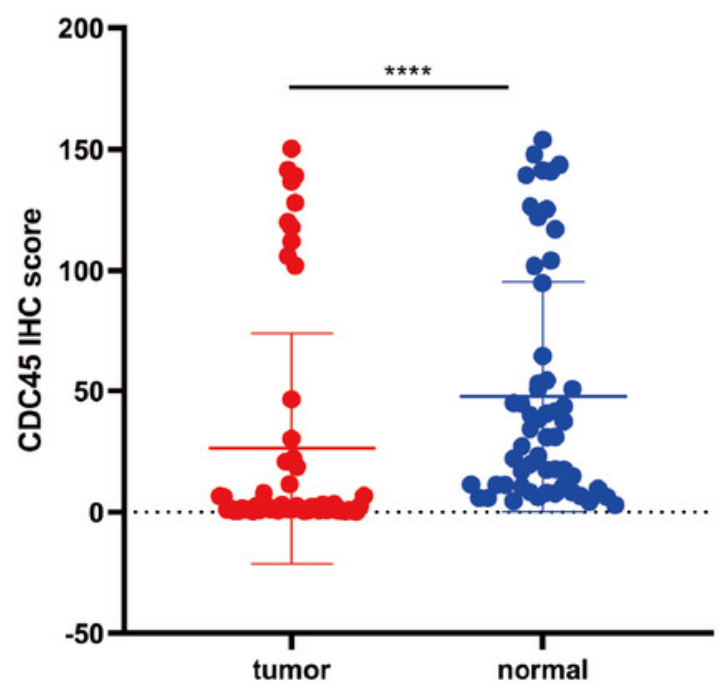

C

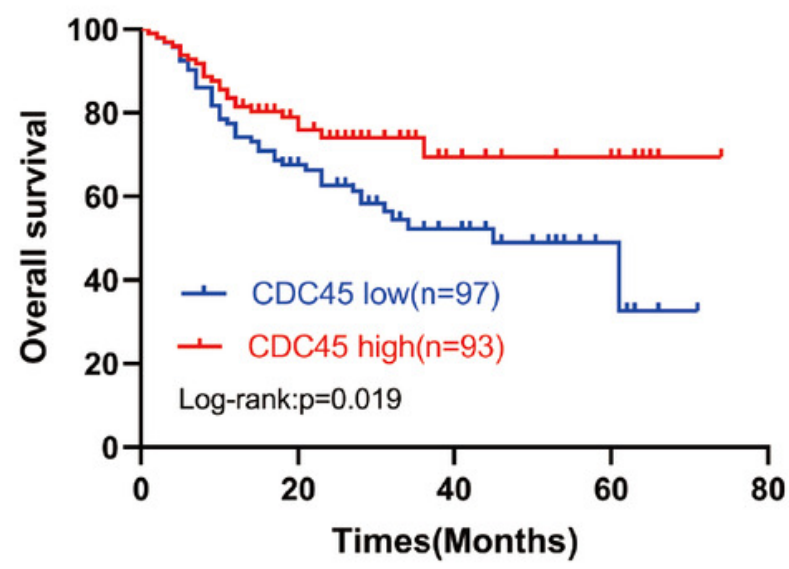




\section{Figure 1}

Enrichment analysis and PPI network construction among DEGs.

(A) Distributions of up- and downregulated DEGs are shown in the volcano plot. The blue colour represents the downregulated genes, while the yellow colour represents the downregulated genes. (B) The heat map shows the top 20 significant DEGs with positive and negative correlations with CDC45. (C) The 30 most enriched GO terms of the DEGs were obtained in HCC patients. (D) Top 30 enriched KEGG pathways of the DEGs are shown in the bar plot. 
A

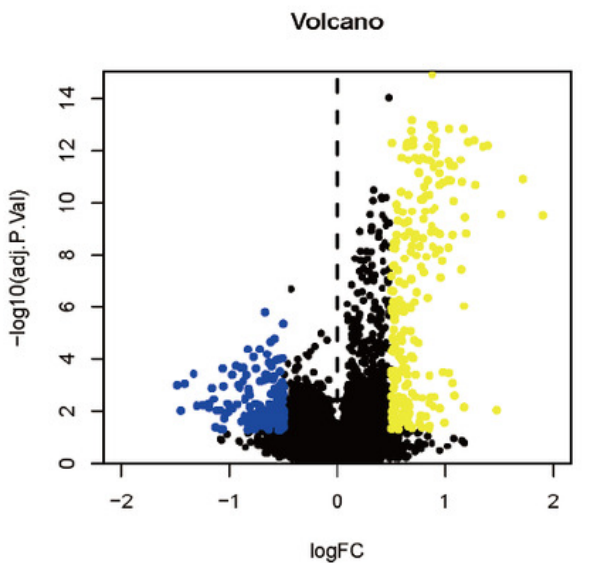

B

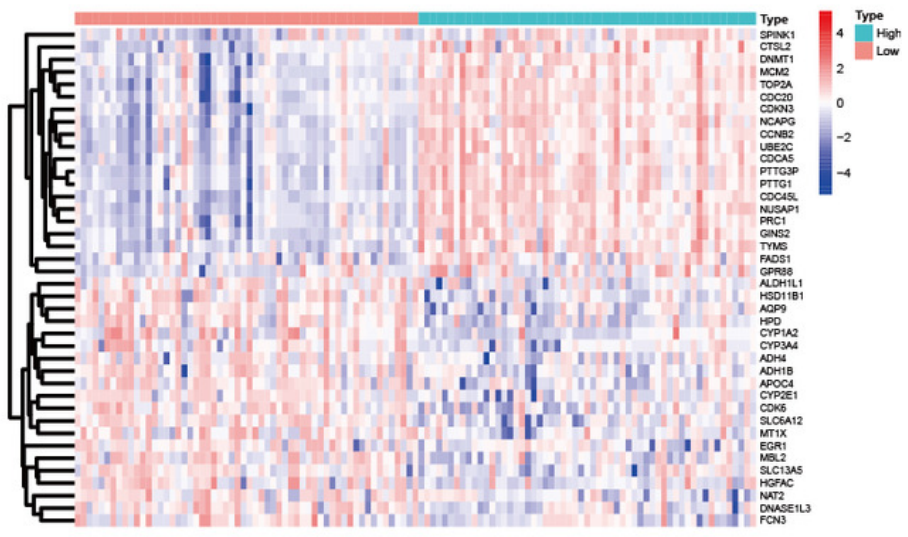

C



D

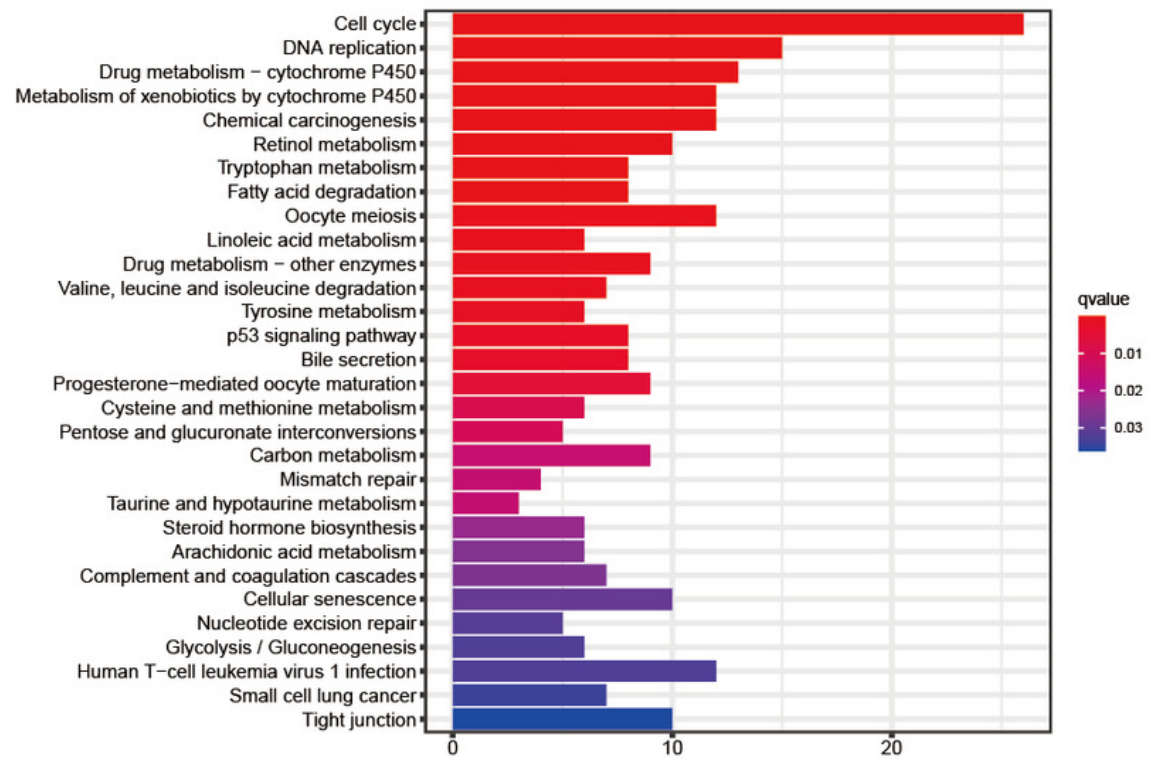


Figure 2

PPI network construction of DEGs and module analysis.

(A) Yellow nodes among the PPI network represent upregulated genes and blue nodes for downregulated genes, and purple nodes for CDC45. (B) Module 1 of the PPI network. (C) Module 2 of the PPI network. (D) Module 3 of the PPI network.
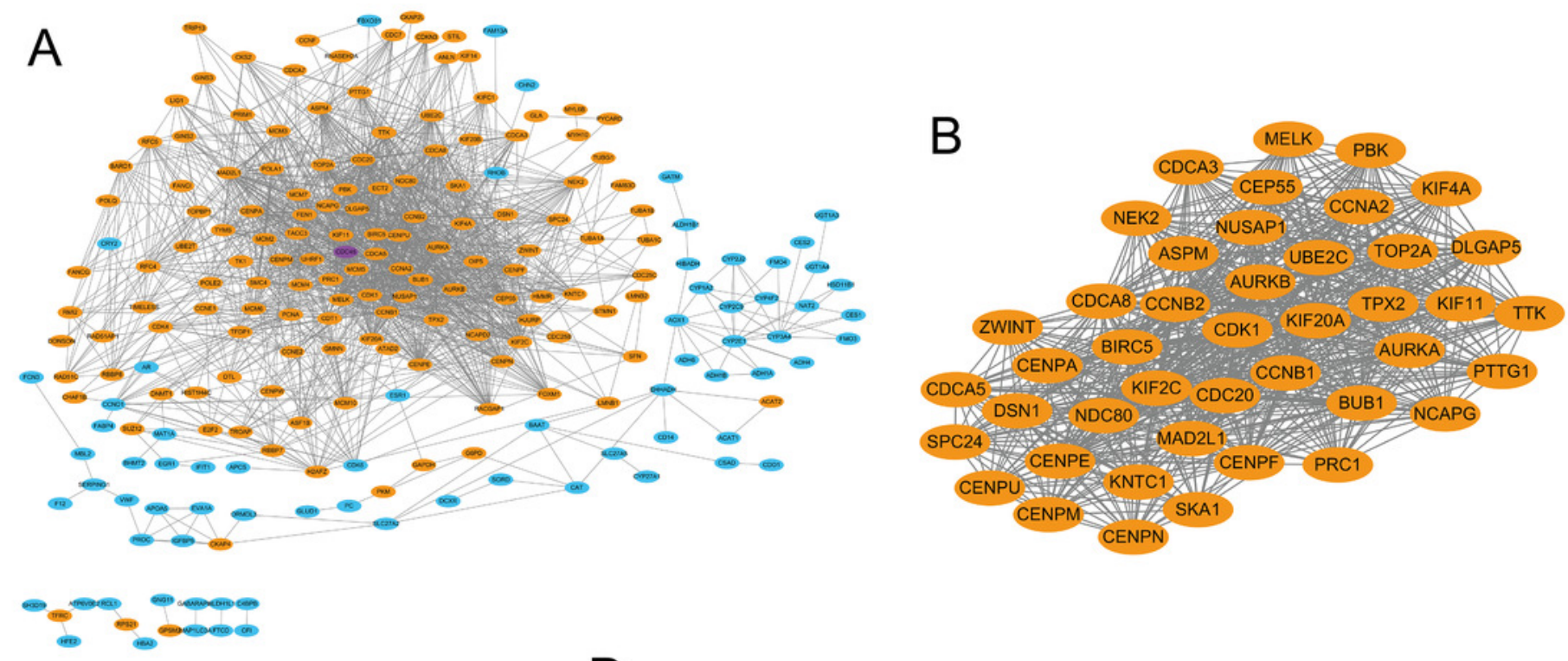

$\mathrm{C}$

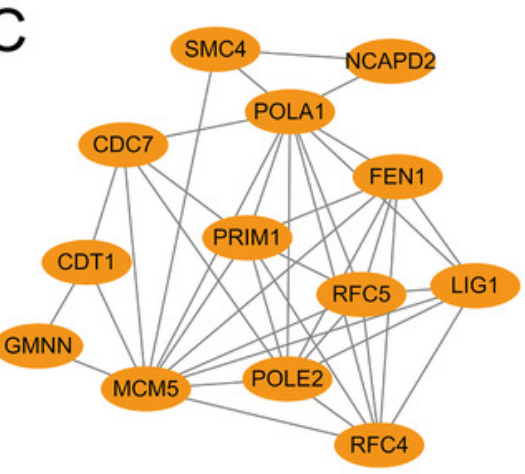

\section{D}

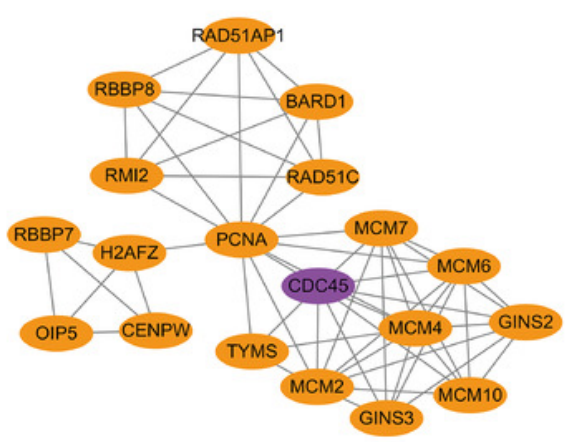

